University of Nebraska - Lincoln

DigitalCommons@University of Nebraska - Lincoln

$1-2015$

\title{
Elasticity of Differentiated and Undifferentiated Human Neuroblastoma Cells Characterized by Atomic Force Microscopy
}

\author{
Shijia Zhao \\ University of Nebraska-Lincoln, s-szhao3@unl.edu \\ Alexander B. Stamm \\ University of Nebraska-Lincoln, alexstamm@gmail.com \\ Jeong Soon Lee \\ University of Nebraska-Lincoln, jlee12@unl.edu \\ Alexei Gruverman \\ University of Nebraska-Lincoln, agruverman2@unl.edu \\ Jung Yul Lim \\ University of Nebraska-Lincoln, jlim4@unl.edu
}

See next page for additional authors

Follow this and additional works at: https://digitalcommons.unl.edu/mechengfacpub

Part of the Biological and Chemical Physics Commons, and the Biophysics Commons

Zhao, Shijia; Stamm, Alexander B.; Lee, Jeong Soon; Gruverman, Alexei; Lim, Jung Yul; and Gu, Linxia, "Elasticity of Differentiated and Undifferentiated Human Neuroblastoma Cells Characterized by Atomic Force Microscopy" (2015). Mechanical \& Materials Engineering Faculty Publications. 114.

https://digitalcommons.unl.edu/mechengfacpub/114

This Article is brought to you for free and open access by the Mechanical \& Materials Engineering, Department of at DigitalCommons@University of Nebraska - Lincoln. It has been accepted for inclusion in Mechanical \& Materials Engineering Faculty Publications by an authorized administrator of DigitalCommons@University of Nebraska Lincoln. 


\section{Authors}

Shijia Zhao, Alexander B. Stamm, Jeong Soon Lee, Alexei Gruverman, Jung Yul Lim, and Linxia Gu 


\title{
Elasticity of Differentiated and Undifferentiated Human Neuroblastoma Cells Characterized by Atomic Force Microscopy
}

\author{
Shijia Zhao, ${ }^{1}$ Alex Stamm, ${ }^{2}$ Jeong Soon Lee, ${ }^{1}$ \\ Alexei Gruverman, ${ }^{2}$ Jung Yul Lim, ${ }^{1,3}$ and Linxia Gu${ }^{1}$ \\ 1 Department of Mechanical \& Materials Engineering, \\ University of Nebraska-Lincoln, Lincoln, NE 68588 \\ 2 Department of Physics \& Astronomy, \\ University of Nebraska-Lincoln, Lincoln, NE 68588 \\ 3 The Graduate School of Dentistry, \\ Kyung Hee University Seoul 130-701, South Korea \\ Corresponding author - L. Gu, lgu@unl.edu
}

\begin{abstract}
Human neuroblastoma (SH-SY5Y) cells, with its ability to differentiate into neurons, have been widely used as the in vitro cell culture model for neuroscience research, especially in studying the pathogenesis of Parkinson's disease (PD) and developing therapeutic strategies. Cellular elasticity could potentially serve as a biomarker to quantitatively distinguish undifferentiated and differentiated SH-SY5Y cells. The goal of this work is to characterize the retinoic acid (RA) induced alternations of elastic properties of SH-SY5Y cells using atomic force microscopy (AFM). The elasticity was measured at multiple points of a single cell. Results have shown that the differentiation of SH-SY5Y cell led to a larger elastic modulus, which is three times more than that of undifferentiated cells. A higher indentation rate applied during AFM measurements led to a larger elastic modulus of the cell. This work provides new insights into the differentiation process identified by the elasticity marker, which could be extended to investigate the function, health and ageing of cells.
\end{abstract}

Keywords: Elasticity, human neuroblastoma SH-SY5Y cells, differentiation, retinoic acid

\section{Introduction}

Parkinson's disease (PD), the second most common neurological disease, is age-related progressive loss of neurons in the brain. ${ }^{1}$ The neuronal degradation was extensively studied through in vitro cell culture models for better understanding the mechanism of PD. 
Specifically, the human neuroblastoma SH-SY5Y cell line, derived from human sympathetic neuronal tissue, ${ }^{2}$ has been commonly used to investigate the pathogenesis of neuronal degeneration ${ }^{3}$ and apoptosis. ${ }^{4}$ Various neurogenic soluble factors, such as brain derived neurotrophic factor, ${ }^{5}$ norepinophrine, and retinoic acid (RA), were used to differentiate SHSY5Y cell into a mature neuronal phenotype with extensive neurite outgrowth. ${ }^{6}$ Cells were generally considered to be differentiated based on the altered morphology only. ${ }^{7}$ To fully understand the differentiation process, it requires further biochemical and biomechanical characterization of SH-SY5Y cells. ${ }^{8}$

Cellular elasticity, a potential biomechanical indicator, provide quantitative information for inferring cellular function and health. ${ }^{9}$ It has served as the biomarker for detecting the abnormal behavior of cancerous cells, ${ }^{10}$ genetically defected red blood cells, ${ }^{11}$ the ageing process of the human epithelial cells, ${ }^{12}$ the shear-induced deformation of bovine endothelial cells, ${ }^{13}$ etc. Various techniques, including micropipette aspiration, ${ }^{14}$ the biomembrane force probe, ${ }^{15}$ optical tweezers, ${ }^{16}$ magnetic tweezers, ${ }^{17}$ and atomic force microscope (AFM) ${ }^{18}$ were used for probing the elasticity of cells.

In this work, nanoscale indentations on both differentiated and undifferentiated SHSY5Y cells were conducted using AFM. The experimentally obtained force curves at multiple points of a single cell were fitted to a Sneddon contact model to obtain the elastic moduli. The cellular heterogeneity as well as the elasticity for both differentiated and undifferentiated SH-SY5Y cells were characterized. The effect of indentation rate on the measured elastic moduli was also studied.

\section{Material and Methods}

Human neuroblastoma SH-SY5Y cells (ATCC) were cultured in high glucose Dulbecco's modified Eagle's media (DMEM) supplemented with 10\% fetal bovine serum and 1\% penicillin-streptomycin (Invitrogen). The cells were maintained in a $5 \% \mathrm{CO}_{2}$ humidified incubator at $37^{\circ} \mathrm{C}$. The pre-coating of 18 -mm-diameter glass coverslips were performed in 12-well plates with Porcogen collagen I solution $(50 \mu \mathrm{g} / \mathrm{mL})$ (SunMax Biotechnology) and incubated for $1 \mathrm{~h}$ at room temperature under a culture hood. After $1 \mathrm{~h}$, the collagen I solution was aspirated. The collagen I coated coverslips were then washed three times with phosphate-buffered saline (PBS, Sigma). This pre-coating process was completed immediately before cell culture. For seeding cells, SH-SY5Y cells were treated with $0.25 \%$ Ethylenediaminetetraacetic acid (EDTA, Invitrogen) and harvested in a $15 \mathrm{~mL}$ centrifuge tube. After centrifugation at $1200 \mathrm{rpm}$ for $5 \mathrm{~min}$, supernatant was aspirated and then fresh media was replaced. The collected cells were counted with hemocytometer and seeded on the collagen I coated glass coverslips with a seeding density of $5 \times 10^{3}$ cells/ coverslip. This low seeding density allows cells separate from each other for convenient characterization of single SH-SY5Y cell. To obtain undifferentiated and differentiated 
cells, SH-SY5Y cells were treated with the same media (DMEM supplemented with 10\% fetal bovine serum and $1 \%$ penicillin-streptomycin) further supplemented with 0 or 10 $\mu \mathrm{g} / \mathrm{mL}$ RA for seven days. The media were refreshed every two days, and on the 7th day, the cells were separated from each other and ready for the AFM test. For confocal immunofluorescence imaging, cultured cells were washed with PBS, fixed with $4 \%$ paraformaldehyde, and permeabilized with $0.1 \%$ Triton X-100 solution for $15 \mathrm{~min}$. They were then incubated for $45 \mathrm{~min}$ with $1 \%$ bovine serum albumin solution and following with rhodamine phalloidin (Invitrogen) for $30 \mathrm{~min}$ to stain actin. After washing, nucleus was double-stained with 4',6-diamidino-2-phenylindole (DAPI, Invitrogen) for one min. Stained actin and nuclei were then observed under a fluorescence microscope (Leica DMI 4000B, Germany).

A commercial AFM system (MFP-3D Stand Alone, Asylum Research) was used in this work. The scanning range of this AFM is $90 \mu \mathrm{m} \times 90 \mu \mathrm{m}$ at XY plane and $15 \mu \mathrm{m}$ along Z direction. Silicon nitride cantilevers (spring constant: 0.02 N/m, Olympus TR400PB) with tip radii of $42 \pm 12 \mathrm{~nm}$ were used for cellular imaging and indentation in culture medium. Prior to conducting indentations on cells, the deflection sensitivity of cantilever was calibrated by taking a force curve via indentation on the standard bare glass slide, and a trigger level of $0.45 \mathrm{~V}$ was used in this calibration. For the experiments presented here, the deflection sensitivity was set as $125.29 \mathrm{~nm} / \mathrm{V}$. The spring constant was then calibrated as $0.02246 \mathrm{~N} / \mathrm{m}$ using thermal tune method. ${ }^{19}$ Before AFM experiments, the cells were first observed and imaged under an optical microscope (Leica), and the cell sizes were estimated. During AFM experiments, the coverslip with cells was first mounted on a Petri dish and immersed in DMEM with HEPES buffer. They were then mounted on the AFM stage. Under the guide of the optical microscope integrated in AFM, both the tips and cells could be observed. The indentations were conducted on a square area of $12 \times 12 \mathrm{~m}^{2}$ at the central portion of the cell, based on the estimated cell size of around $50 \times 20 \mathrm{~m}^{2}$ for differentiated cell. Total $36(6 \times 6)$ indentations were conducted with a lateral distance of $2 \mu \mathrm{m}$. The force indentation curve at each point was obtained using a force control mode, which is manipulated by defining a trigger level of $0.4 \mathrm{~V}$, i.e., an equivalent indentation force up to $1.1 \mathrm{nN}$. The indentation rate was set as $5.95 \mu \mathrm{m} / \mathrm{s}$. To investigate the effect of indentation rate on measured cellular elasticity, a smaller indentation rate of $0.5 \mu \mathrm{m} / \mathrm{s}$ was used. Both indentation rates were consistent with the range $(0.1-10 \mu \mathrm{m} / \mathrm{s})$ used for a single cortical neurons. ${ }^{20}$ During each indentation cycle, the displacement and the deflection of the cantilever were monitored and recorded. All AFM experiments on the cells were performed in media at room temperature.

The obtained deflection and displacement data were used to calculate the elastic modulus of cell. Based on Hooke's law, the indentation force $F(\mathrm{nN})$ was calculated as the product of cantilever spring constant $k(\mathrm{~N} / \mathrm{m})$ and deflection of the cantilever $\mathrm{d}(\mathrm{nm})$ as $F=k \cdot d$. The actual indentation depth $\delta(\mathrm{nm})$ was obtained by measuring the difference between the displacement $z(\mathrm{~nm})$ and deflection $d(\mathrm{~nm})$ as $\delta=z-d$. The deflection-displacement curve 
was then converted into a force-indentation curve. The Sneddon contact model for a conical tip ${ }^{21}$ was adopted to fit the approaching portion of the force curve:

$$
F=\frac{2}{\pi} \cdot \frac{E}{1-v^{2}} \cdot \delta^{2} \cdot \tan \alpha
$$

where $E$ is the elastic modulus of the sample $(\mathrm{Pa}), v$ is the Poisson's ratio $(0.5)$ and $\alpha$ is the half cone angle of the conical tip $\left(35^{\circ}\right)$. The least-squares method was used to fit the experimental indentation curves with the non-linear Sneddon model and, therefore, to derive the cellular elastic modulus.

\section{Results}

After SH-SY5Y cells were differentiated with RA exposure for seven days, cells were visualized using optical microscope (Leica); meanwhile, undifferentiated cells were grown the same way but without RA (Figure 1). The spindle shape of cell body and the neurite outgrowth for the differentiated cells could be observed. In the immunofluorescent imaging of actin (Figure 2), well-developed actin microfilaments could be observed for RA-treated differentiated SH-SY5Y cells. On the other hand, for undifferentiated cells actin staining was rather diffused and filament-like structure was seldom seen.

After the indentations on differentiated SH-SY5Y cell, the local elastic moduli were obtained. The histograms for cellular elastic moduli of both differentiated and undifferentiated cells were depicted in Figure 3. It is clear that the frequency distribution of differentiated cell is towards larger elastic moduli, compared with the undifferentiated cell. By averaging the elastic moduli at all points, the elasticity of the differentiated SH-SY5Y cell was $1368.8 \pm 773.9 \mathrm{~Pa}$ and $448.9 \pm 261.5 \mathrm{~Pa}$ for undifferentiated cells.

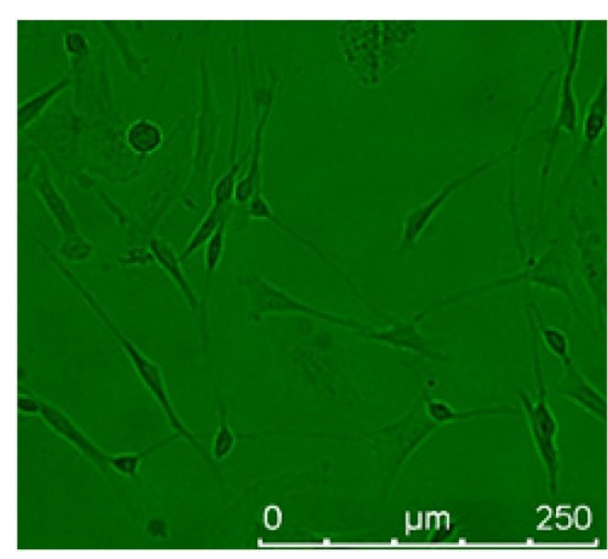

(a)

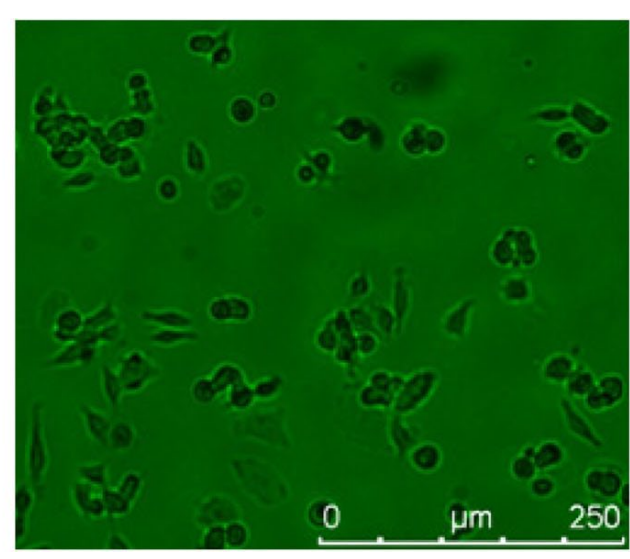

(b)

Figure 1. Optical microscopic images of differentiated (a) and undifferentiated (b) SH-SY5Y cells. 


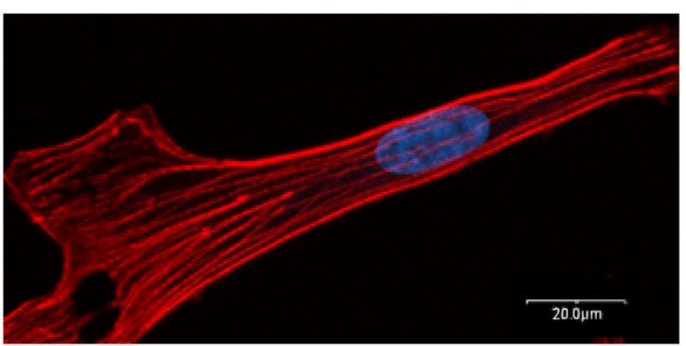

(a)

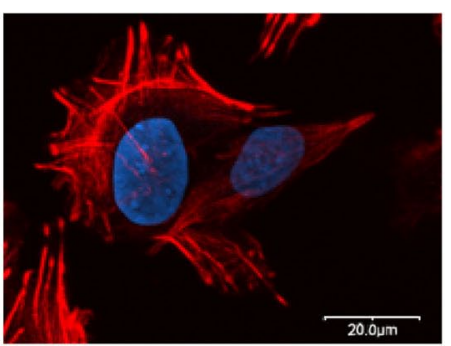

(b)

Figure 2. Immunofluorescent images of actin cytoskeletons (red) and nucleus (blue) for differentiated (a) and undifferentiated (b) SH-SY5Y cells (scale bar $=20.0 \mu \mathrm{m}$ ).

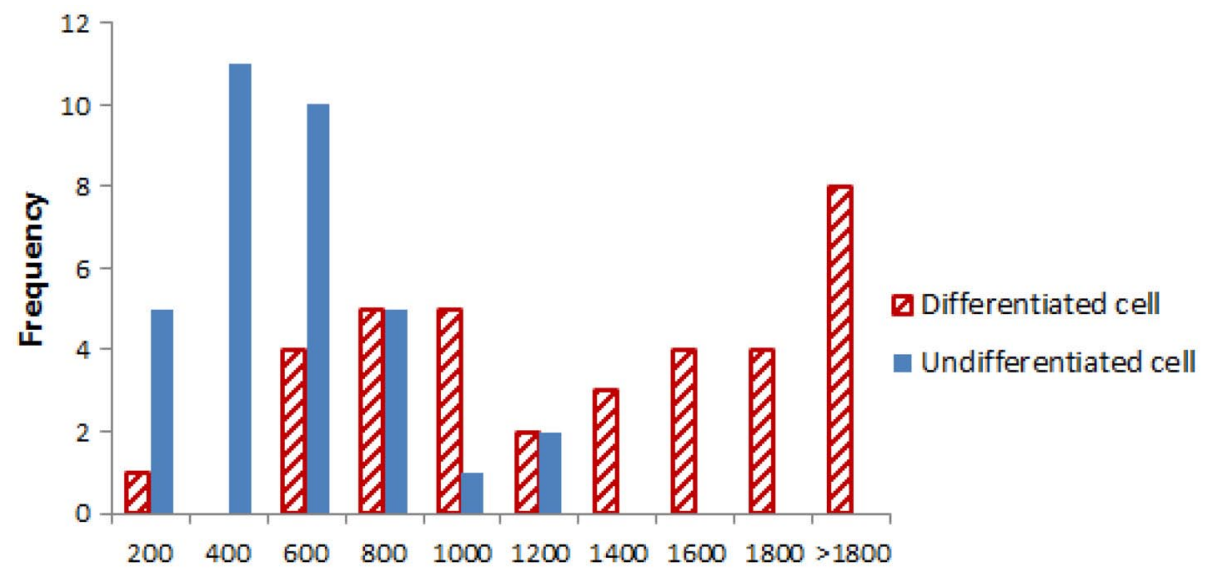

\section{Elastic modulus (Pa)}

Figure 3. The histogram of elastic moduli for both differentiated and undifferentiated SH-SY5Y cells.

A larger standard deviation was expected due to the inherent heterogeneity of both differentiated and undifferentiated cells. This is consistent with other cellular studies. ${ }^{22}$ The average elastic modulus of differentiated cell is much larger than the modulus for undifferentiated cell. To calculate the statistically significant difference between two mean elastic moduli of both undifferentiated and differentiated cells, a $z$-test instead of $t$-test was used considering both dataset sizes are larger than $30 .{ }^{23}$ The null hypothesis is that the difference between two means is $800 \mathrm{~Pa}$, while the alternative hypothesis is that the difference is larger than $800 \mathrm{~Pa}$. The test statistic was calculated using the formula below. ${ }^{23}$

$$
z=\frac{\bar{y}_{1}-\bar{y}_{2}-\left(\mu_{1}-\mu_{2}\right)_{0}}{\sqrt{\frac{\sigma_{1}^{2}}{n_{1}}+\frac{\sigma_{2}^{2}}{n_{2}}}}
$$

where $\bar{y}_{1}$ and $\bar{y}_{2}$ are the calculated means of elastic modulus for one differentiated and one undifferentiated cell, respectively; $\left(\mu_{1}-\mu_{2}\right)_{0}=800 ; \sigma_{1}^{2}$ and $\sigma_{2}^{2}$ are the calculated variances 


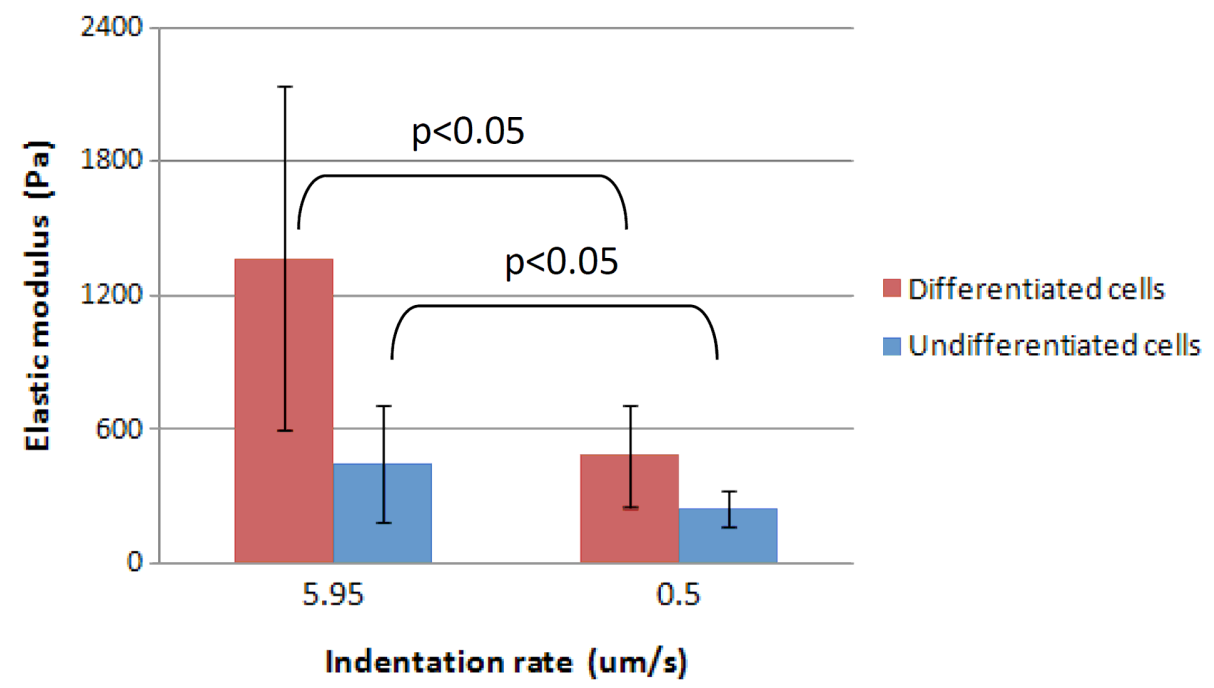

Figure 4. The role of indentation rates on the elastic modulus.

for two cells, respectively; while $n_{1}$ and $n_{2}$ are their respective sample sizes. The significance level was chosen as 0.05 . The calculated $z$ score statistic is $z=0.88>z_{0.05}=0.48$, indicating that the null hypothesis was rejected and the mean value of the differentiated cell elasticity is larger than the undifferentiated cell by at least $800 \mathrm{~Pa}$ from the statistical view at indentation rate of $5.95 \mu \mathrm{m} / \mathrm{s}$.

The indentation rate adopted in the reported AFM tests varied, and its impact on the cellular elasticity was controversial. ${ }^{8,24}$ Herein two indentation rates, i.e., 5.95 and $0.5 \mu \mathrm{m} / \mathrm{s}$ were compared for both undifferentiated and differentiated cells to quantify the effect of indentation rate on the elasticity of SH-SY5Y cells (Figure 4). For a smaller indentation rate of $0.5 \mu \mathrm{m} / \mathrm{s}$ the elastic moduli were calculated as 249.2 $\pm 81: 3$ Pa for undifferentiated cells, and 483.2 $\pm 226.7 \mathrm{~Pa}$ for differentiated cells, respectively. Both were much smaller $(p<0.05)$, than the ones under the indentation rate of $5.95 \mu \mathrm{m} / \mathrm{s}$. The mean elastic modulus for differentiated cell was increased by $183 \%$, while it is $80 \%$ for undifferentiated cell when indentation rate varied from 0.5 to $5.95 \mu \mathrm{m} / \mathrm{s}$.

\section{Discussion}

It is well acknowledged that the function of a cell is regulated by its structure, which varies during its adhesion, proliferation, differentiation, as well as during pathogenesis such as oxidative stress and attack of viruses and parasites. ${ }^{18}$ We have observed the distinct morphology variations (Figure 2) after the differentiation of SH-SY5Y cells induced by RA. The differentiated cells exhibited neurite extension compared to the undifferentiated cells. The RA-induced differentiation in SH-SY5Y cells were generally evaluated by 
their immunoreactivity of different neuronal markers ${ }^{6}$ and the resistance to hyperthermic stress. ${ }^{25}$ Although other types of cells were reported to have varied mechanical properties and cytoskeleton reorganization during differentiation or ageing process, ${ }^{12,26,27}$ remarkably, the mechanical properties of SH-5Y5Y cells are barely characterized in the literature.

The pyramid probe was adopted in this work for obtaining higher resolution map of local elasticity. The elasticity of SH-SY5Y cells was calculated by fitting the classical Sneddon model to the approaching portion of the AFM force curves. ${ }^{9,10,28}$ It is a widely accepted model for estimating the local elasticity especially when the indentations are controlled under small deformation. ${ }^{18}$ This model is justified considering the small indentation depth ( $<10 \%$ of cellular thickness) and the comparative nature of our work between undifferentiated and differentiated SH-SY5Y cells. ${ }^{29}$

We hypothesized here that cellular elasticity could be a biomarker for the differentiation of SH-SY5Y cells, and used AFM to quantify the elasticity of both differentiated and undifferentiated SH-SY5Y cells. Results have shown that the differentiation of SH-SY5Y cell demanded a larger elastic modulus than the undifferentiated one, which demonstrated a similar trend as the differentiation of other cell types, such as mammalian myocytes $^{26,30}$ and mouse embryonic stem cells. ${ }^{31}$ This indicated that the ability of differentiated SH-SY5Y cells to resist elastic deformation increased compared to undifferentiated cells, which can be correlated to the changes of subcellular structures. The cytoskeleton, especially the actin cytoskeleton which interacts with cell membranes, is an important reflection of cellular structure organization. It was observed in Figure 2 that the differentiated cell seems to possess a well-developed network of actin microfilaments than undifferentiated cell. The lack of the well-developed actin filaments or their bundles in the undifferentiated cell leads to a weak cytoskeleton structure, which could explain the reduced elastic moduli of the undifferentiated cell when comparing to its differentiated counterpart. This also implies that the undifferentiated SH-SY5Y cells are prone to deformation and related migration. ${ }^{32}$

Currently there is no standard AFM protocol specifying the indentation rate for the cellular testing, which could post difficulty to compare between observations from various research group. ${ }^{8,24}$ In this work, we have illustrated the effect of indentation rate on the measured elasticity of both undifferentiated and differentiated SH-SY5Y cells. It is clear that the higher indentation rate would induce the larger elastic modulus of cell. This could be explained by the viscoelastic nature of cellular materials, ${ }^{33,34}$ i.e., the potential energy is difficult to dissipate with a faster indentation. The actin network within the differentiated cell - better developed than the undifferentiated one (Figure 2) - was also correlated to the different levels of viscous response. This agreed with the observation by Ketene et al. ${ }^{35}$ Moreover, the results of our indentation rate tests consistently showed that the differentiated SH-SY5Y cell is stiffer than the undifferentiated counterpart. 


\section{Conclusion}

Our work has demonstrated that RA-induced neuronal differentiation not only changed the morphology of SH-SY5Y cell, but also increased the elasticity of the cell body. Both the differentiated and undifferentiated SH-SY5Y cells showed the heterogeneous mechanical properties on the surface of one single cell. It was suggested to check the indentation rate when comparing results from various resources. These results might suggest using elasticity as a potential biomarker for cell differentiation or cell maturation of SH-SY5Y cells. These findings may aid the studies designed to investigate the pathogenesis of PD using SH-SY5Y cell lines as the in vitro model, where the biomechanical properties can be used to identify the neuronal differentiation stage of SH-SY5Y cells. Since the PD is an ageingrelated disease, the ageing impact on the SH-SY5Y cells will be incorporated into the future investigations.

Acknowledgments - This project is partially funded by the National Science Foundation under grant No. 1254095, and by a UNL Research Council Interdisciplinary Grant.

\section{References}

1. Kontakos, N, Stokes J, Monograph series on aging-related diseases: XII. Parkinson's disease - Recent developments and new directions, Chronic Dis Can 20(2):58-76, 1999.

2. Sousa, SR et al., Expression and Pharmacology of Endogenous Ca-v Channels in SH-SY5Y Human Neuroblastoma Cells, PLoS One 8(3), 2013.

3. Park, YJ, Jang Y, Kwon YH, Protective effect of isoflavones against homocysteine-mediated neuronal degeneration in SH-SY5Y cells. Amino Acids 39(3):785-794, 2010.

4. Chang, RC et al., Involvement of double-stranded RNA-dependent protein kinase and phosphorylation of eukaryotic initiation factor-2 alpha in neuronal degeneration, J Neurochem 83(5):1215-1225, 2002.

5. Encinas, M et al., Sequential treatment of SH-SY5Y cells with retinoic acid and brain-derived neurotrophic factor gives rise to fully differentiated, neurotrophic factor-dependent, human neuron-like cells, J Neurochem 75(3):991-1003, 2000.

6. Cheung, YT et al., Effects of all-trans-retinoic acid on human SH-SY5Y neuroblastoma as in vitro model in neurotoxicity research, Neurotoxicology 30(1):127-135, 2009.

7. Constantinescu, R et al., Neuronal differentiation and long-term culture of the human neuroblastoma line SH-SY5Y, J Neural Transm Suppl 72:17-28, 2007.

8. Kiss, R et al., Elasticity of human embryonic stem cells as determined by atomic force microscopy, J Biomech Eng-Trans ASME 133(10), 2011.

9. Mustata, M, Ritchie K, McNally HA, Neuronal elasticity as measured by atomic force microscopy, J Neurosci Meth 186(1):35-41, 2010.

10. Li, QS et al., AFM indentation study of breast cancer cells, Biochem Biophys Res Commun 374(4):609-613, 2008. 
11. Mohandas, N, Evans E, Mechanical properties of the red cell membrane in relation to molecular structure and genetic defects, Annu Rev Biophys Biomol Struct 23:787-818, 1994.

12. Berdyyeva, TK, Woodworth CD, Sokolov I, Human epithelial cells increase their rigidity with ageing in vitro: Direct measurements, Phys Med Biol 50(1):81-92, 2005.

13. Ohashi, $\mathrm{T}$ et al., Experimental and numerical analyses of local mechanical properties measured by atomic force microscopy for sheared endothelial cells, Biomed Mater Eng 12(3):319327, 2002.

14. Evans, E, Mohandas N, Leung A, Static and dynamic rigidities of normal and sickle erythrocytes. Major influence of cell hemoglobin concentration, J Clin Invest 73(2):477- 488, 1984.

15. Heinrich, $\mathrm{V}$ et al., Elastic thickness compressibilty of the red cell membrane. Biophys J 81(3):1452-1463, 2001.

16. Sleep, J et al., Elasticity of the red cell membrane and its relation to hemolytic disorders: An optical tweezers study. Biophys J 77(6):3085-3095, 1999.

17. Bausch, AR, Moller W, Sackmann E, Measurement of local viscoelasticity and forces in living cells by magnetic tweezers, Biophys J 76(1 Pt 1):573-579, 1999.

18. Kuznetsova, TG et al., Atomic force microscopy probing of cell elasticity, Micron 38(8):824833, 2007.

19. Hutter, JL, Bechhoefer J, Calibration of Atomic-Force Microscope Tips, Rev Sci Instruments 64(7):1868-1873, 1993.

20. Bernick, KB et al., Biomechanics of single cortical neurons, Acta Biomaterialia 7(3):1210-1219, 2011.

21. Sneddon, IN, The relation between load and penetration in the axisymmetric Boussinesq problem for a punch of arbitrary profile, Int J Eng Sci 3(1):47-57, 1965.

22. Xu,WW et al., Cell stiffness is a biomarker of the metastatic potential of ovarian cancer cells, PLoS One 7(10), 2012.

23. Dowdy, S, Weardon S, Chilko D, Statistics for Research, John Wiley \& Sons, Hoboken, New Jersey, 2004.

24. Chang, IY-T, Study of T cell activation and migration at the single-cell and single-molecule level, Massachusetts Institute of Technology, 2011, p. 184.

25. Cheng, L et al., Human neuroblastoma SH-SY5Y cells show increased resistance to hyperthermic stress after differentiation, associated with elevated levels of Hsp72, Int J Hyperthermia 27(5):415-426, 2011.

26. Collinsworth, AM et al., Apparent elastic modulus and hysteresis of skeletal muscle cells throughout differentiation, Am J Physiol Cell Physiol 283(4):C1219-C1227, 2002.

27. Lieber, SC et al., Aging increases stiffness of cardiac myocytes measured by atomic force microscopy nanoindentation, Am J Physiol Heart Circ Physiol 287(2):H645-H651, 2004.

28. Guo, Q et al., Characterization of cell elasticity correlated with cell morphology by atomic force microscope, J Biomech 45(2):304-309, 2012.

29. Prabhune, $\mathrm{M}$ et al., Comparison of mechanical properties of normal and malignant thyroid cells, Micron 43(12):1267-1272, 2012.

30. Zhang, JS, Kraus WE, Truskey GA, Stretch-induced nitric oxide modulates mechanical properties of skeletal muscle cells, Am J Physiol Cell Physiol 287(2):C292-C299. 
31. Pillarisetti, A et al., Mechanical characterization of mouse embryonic stem cells. Conf Proc IEEE Eng Med Biol Soc 2009: 1176-1179, 2009.

32. Wolf, K et al., Physical limits of cell migration: Control by ECM space and nuclear deformation and tuning by proteolysis and traction force. Journal of Cell Biology 201(7):1069-1084, 2013.

33. Alcaraz, J et al., Microrheology of human lung epithelial cells measured by atomic force microscopy. Biophys J 84(3):2071-2079, 2003.

34. Smith, BA et al., Probing the viscoelastic behavior of cultured airway smooth muscle cells with atomic force microscopy: Stiffening induced by contractile agonist, Biophys J 88(4):2994-3007, 2005.

35. Ketene, AN et al., Actin filaments play a primary role for structural integrity and viscoelastic response in cells, Integr Biol: Quant Biosci Nano to Macro 4(5):540-549, 2012. 